\title{
Invited Paper
}

\section{Systems-Based Framework for Continuing Medical Education and Improvements in Translating New Knowledge into Physicians' Practices}

\author{
R. Van Harrison, $\mathrm{PhD}$
}

\begin{abstract}
Concerns about health care costs and quality are focusing increasing attention on physicians and their continuing medical education (CME). These concerns have produced several calls for "a new definition," "a new vision," "repositioning," "reinventing," and "transforming" CME. However, differences in conceptualizations and vocabularies have introduced appreciable confusion in recommending changes. This article uses a systems-based approach to describe and analyze the processes involved in translating new information into physicians' practices. The article (1) introduces a conceptual framework that links physician learning and performance to systems for information, education, implementation, and regulation in the context of the larger health care system; (2) uses the framework to identify concerns and opportunities for the major types of systems immediately relevant to $C M E$; and (3) uses the framework to suggest broader implications for CME, including the nature of process for changing physicians' practices, needed improvements, priorities in performing research, and implications for CME professionals.
\end{abstract}

Key Words: Continuing medical education (CME), education, implementation, information, systems theory, translating research into practice

Concerns about health care costs and quality are focusing increasing attention on physicians and their continuing medical education (CME). Physicians have a central role in decisions about health care and health care expenditures. In 2000 , personal health care expenditures in the United States totaled over a trillion dollars, with hospital care, physician services, and prescription drugs account-

Dr. Harrison: Professor, Department of Medical Education, and Director, Office of Continuing Medical Education, University of Michigan Medical School, Ann Arbor, Michigan.

This article is based on two presentations made at CME Congress 2004, Toronto, ON, May 16-18, 2004. Some of the concepts evolved from discussions with David A. Davis, MD, during the planning of CME Congress 2004.

Reprint requests: R. Van Harrison, $\mathrm{PhD}$, Department of Medical Education, University of Michigan, G-1103 Towsley Center, Ann Arbor, MI 48109-0201. ing for $73 \%$ of these expenditures.' Physicians are expected to continue to acquire new knowledge to improve health care, with new information becoming available at an increasing rate. However, studies have shown that new information may or may not be implemented into practice. ${ }^{2}$

These concerns have produced several calls for "a new definition," "a new vision," "repositioning," "reinventing," and "transforming" CME. ${ }^{3-10}$ A common theme is that CME must be conceived more broadly than a lecture-based learning experience or a system of credit for learning experiences. Expansive definitions of CME include "all of the learning that takes place in the professional life of the physician." Some prefer the phrase "continuing professional development" to go beyond didactic formats to include self-directed learning and organizational and system factors. "

Although these calls for a broader conceptualization recognize an important need, much of the 


\section{Harrison}

discussion has been unclear. Different authors and speakers use the term with different meanings. Broad definitions of CME tend to overlap with activities of professionally related fields, such as health services research and quality assurance. ${ }^{12,13}$ The confusion in terminology is further complicated by those related disciplines often defining CME narrowly to refer to formal educational activities that are separate from data feedback and other forms of physician learning. ${ }^{14}$

The purpose of this article is to facilitate translating new information into physicians' practices by clarifying the systems and processes involved. The sections of the article address each of the following specific aims:

1. Introduce a systems-based conceptual framework that links physician learning to the larger societal systems and processes involved in translating knowledge into practice.

2. Use the conceptual framework to identify concerns and opportunities for major systems immediately relevant to CME.

3. Use the conceptual framework to suggest broader implications for CME, including future improvements and research.

\section{Conceptual Framework}

\section{Systems Theory}

General systems theory is a conceptual metatheory that focuses on how a system receives inputs (resources and information) from the environment, processes them, and produces outputs into the environment. The approach has been applied to the analysis of living systems at the levels of the cell, individual, group, organization, and society. ${ }^{15}$ The approach helps conceptualize the effects of relationships across these levels, for example, interrelating the actions of individuals and organizations in which they work. ${ }^{16}$

A conceptual approach based on systems theory is useful for describing the interrelationships across the various systems with which physicians interact in the process of translating knowledge into practice. A systems approach does not exclude other theories as the basis for specific interactions. Rather, the systems framework provides a broader context within which specific interactions occur. An extensive analysis of relevant systems is beyond the scope of this article; up to 19 critical subsystems have been proposed to process matter, energy, and information in a system. ${ }^{15}$ However, the approach provides a good foundation for a framework to identify relevant relationships and plan for future change.

\section{Systems of Primary Interest}

Figure 1 illustrates six types of systems of interest in translating new information into physicians' practices. The arrows represent one type of system initiating action on another. To simplify the diagram, arrows are illustrated only for some main actions of interest:

- Health care environment. The larger societal environment provides input (both resources and demands) to all of the other systems and is impacted by them as well.

- Physicians. Physicians are interacting with the other systems as they become aware of new concepts, how to implement them, and actually provide care.

- Information. As new scientific discoveries occur, they are incorporated into a set of systems that store and disseminate new information (e.g., journals, textbooks) to physicians. Related systems store information primarily for retrieval that physicians initiate (e.g., libraries, computerized databases).

- Education. In these systems, experts synthesize new literature, prioritize it, assess what people in practice need to know, and provide curricula in specific formats. Often these systems provide formal CME with credit. Physicians may participate in educational activities determined by others 


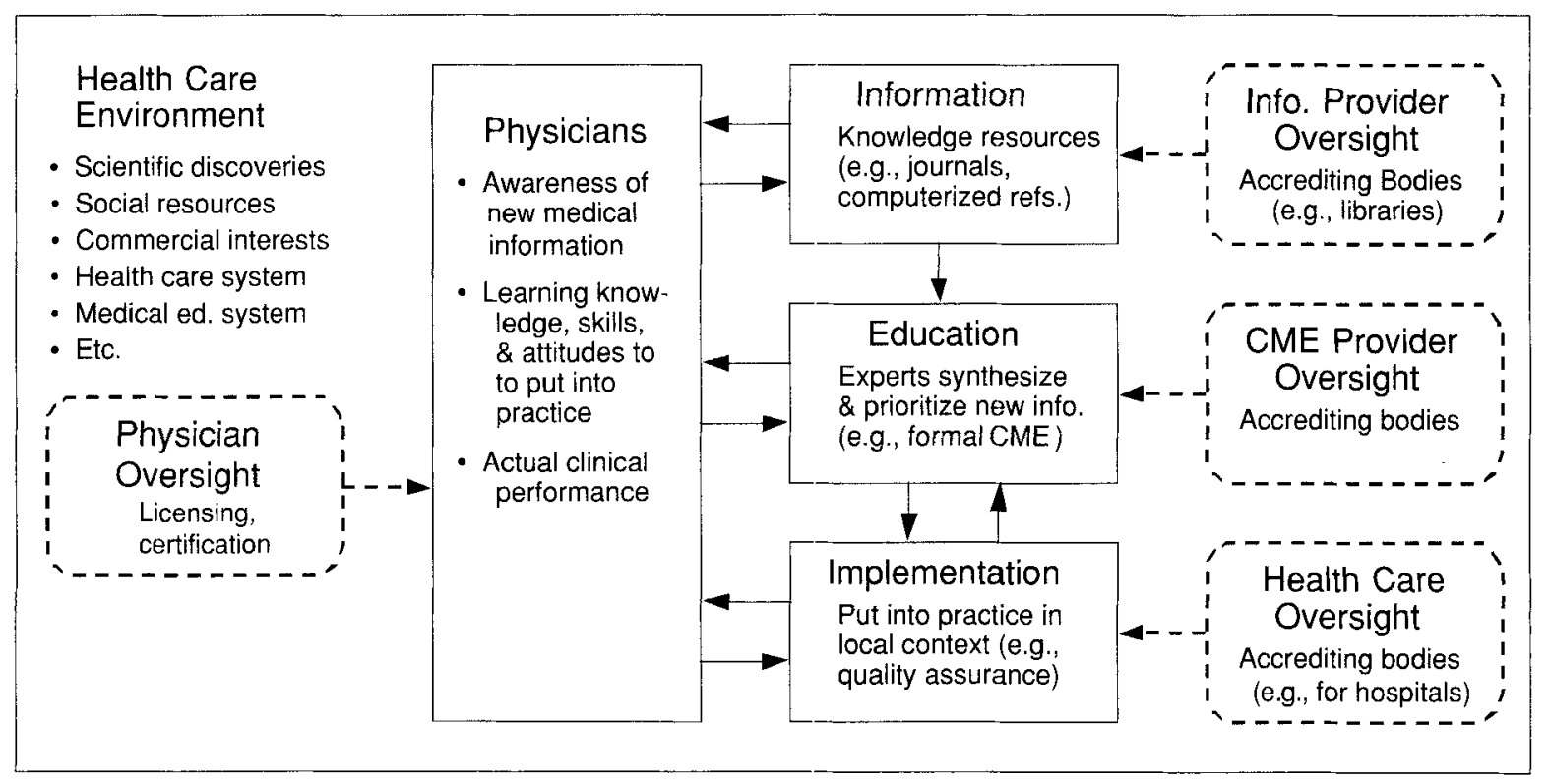

Figure 1 A systems-based conceptual model of the processes for translating new knowledge into physicians' practices.

(e.g., weekly grand rounds topics) or seek out training on specific topics.

- Implementation. Care is actually provided in these systems (e.g., clinics and hospitals). A component of these systems (e.g., quality assurance unit) may initiate action to improve physician performance. Alternatively, physicians may act to change the implementation system to facilitate the provision of care.

- Regulatory oversight. Each of the preceding types of systems has regulatory systems (distinguished by dashed lines in Figure 1) charged with overseeing the appropriateness of the system's activities. Regulatory systems range from bodies that set standards for medical journals to various accrediting, licensing, and credentialing bodies. (Many additional regulatory bodies are relevant to the larger health care environment but are not shown.)

Placing these systems in context with each other highlights the complexity of the overall processes. Each step in the linkage across "information," "education," and "implementation" may be necessary but not in itself sufficient. For example, studies of the failure to perform important care activities have found that often the problem may not lack biologic knowledge but contextual factors in implementation. ${ }^{17}$ The broad framework also highlights the variety of professions involved at various points in the overall process. In addition to medicine, they include information and library science, education, health care administration, quality assurance, and others.

The different systems have their own purposes and agendas. When individuals within a particular system talk about physician learning, they usually do so from a specific perspective. For example, Table 1 presents a set of change processes from the viewpoint of physicians in physicianinitiated learning to translate knowledge into practice. It is based on a model of individuals moving through steps of awareness, agreement, adoption, and adherence. ${ }^{18}$ Presented by each step in Table 1 are activities from the information, education, and implementation systems that are likely to 


\section{Harrison}

Table 1 Practice Change from a Physician's Perspective: Steps and Activities Likely to Facilitate Them

\begin{tabular}{lll}
\hline Change Step* $^{*}$ & Likely Activities $^{\dagger}$ & \\
\hline Awareness & General reading & Informal discussion \\
& CME programs & Marketing message \\
Agreement & Consultation & Influential leaders \\
& Literature search & Problem-based learning \\
Adoption & Simulation & Experience with outcome \\
& Trial, testing & Organize to facilitate \\
Adherence & Routine performance & System changes to facilitate \\
& Ongoing feedback & Monitoring \\
\hline
\end{tabular}

$\mathrm{CME}=$ continuing medical education.

*Based on Pathman et al. ${ }^{18}$

'Based on Burney and Harrison. ${ }^{19}$

facilitate the steps. ${ }^{19}$ This view is consistent with viewing physicians as active learners who reflect on practice. In contrast, Table 2 presents a model for change that organizations commonly use for quality improvement. ${ }^{20}$ In this instance, the organization is acting on the physician. A careful reading of the description shows the organization following a somewhat parallel process of active learning: becoming aware of the issue, agreeing on what changes to make, making (adopting) the change, and adhering based on an evaluation of the result. Although both of these models fall within a larger framework of physician learning and performance, each makes different assumptions about whether physicians are viewed as individuals seeking solutions or as uniform parts of a larger process.

\section{Additional Conceptual Clarifications}

Several additional conceptual clarifications are needed to facilitate discussions of processes within the framework associated with physician learning and the translation of research into practice. Some suggestions for useful clarifications are listed in Table 3. These distinctions address the type of information that is provided, how recently the information became available, the context in which it will be used, the focus in providing it, how it is transmitted, and who will pay for the processes by which information is translated into practice.

\section{Concerns and Opportunities within Major Systems}

The conceptual framework in Figure 1 and additional clarifications in Table 3 help describe trends in the larger health care environment and their implications for concerns and opportunities in each of the other major systems. The following observations illustrate applications of the framework.

\section{Trends in the Health Care Environment}

Major trends in the health care environment that will strongly affect the future of CME include the following:

- Limits on societal resources. Increasing health care costs and societal limits on resources are likely to be the most prominent factors affecting all systems related to the health care environment in the coming decades. ${ }^{21}$

- Increasing oversight. Concerns about health care costs will result in increasing oversight by third-party payers and others to 
Table 2 Change from an Organizational Perspective: Plan-Do-Check-Act Cycle for Continuous Quality Improvement

\begin{tabular}{ll}
\hline Plan & Identify problem, analyze cause(s), develop action plan (Problem involves physician? \\
& New information needed?) \\
Do & Implement action plan (provide education in conjunction with other actions) \\
Check & Evaluate results (reassess physician performance) \\
Act & Redesign as necessary, standardize the process (e.g., consider periodic feedback) \\
\hline
\end{tabular}

The Plan-Do-Check-Act Cycle is also referred to as the Deming Wheel. ${ }^{20}$

ensure that costs are contained without sacrificing quality.

- Increasing rate of new information. Continued investment in research by federal and private resources and increasingly efficient research technologies are likely to introduce changes in health care at an increasing rate.

- Advances in technology. The organization of care is likely to change as electronic databases are integrated and other technology improvements make possible more sophisticated coordination of care.

These trends will fuel increasing societal debate about how resources should be used, what is worth the cost, and who should pay for it. As health care costs consume more of our gross domestic product, physicians' actions in authorizing expenditures will come under increasing scrutiny, oversight, and regulation from the public and private groups.

Table 3 Some Useful Conceptual Clarifications Related to Processes for Translating New Knowledge into Physicians' Practices

\begin{tabular}{ll}
\hline Types of information & Biologic (disease) \\
& Psychosocial (patient) \\
Organizational (operations) & Financial (resources, cost-effectiveness) \\
& Simple cognitive change (e.g., improved drug) \\
& Cognitive and operational change \\
Context of new information & (e.g., outpatient LMWH) \\
& Complex changes (e.g., SARS epidemic) \\
& New (1-2 yr) (e.g., "update" CME course) \\
& Core (e.g., residency training, board review course) \\
& Basic (e.g., remedial course) \\
Information categories & Research report \\
& Literature review \\
& Prioritization for practice \\
Use for information & Fact for reference \\
& Live (synchronous) to individual or group \\
& Stored (asynchronous): print, Internet file, audiotape \\
Methods of transmitting information & Physician \\
& Health care institution \\
Who benefits enough to pay for translation process & Third-party payer \\
& Commercial company \\
& Government \\
\hline
\end{tabular}

$\mathrm{CME}=$ continuing medical education; LMWH = low-molecular-weight heparin; SARS = severe acute respiratory syndrome. 


\section{Effects on Physicians}

These societal trends are likely to have several effects on physicians:

- Time pressure. Clinical and administrative workloads of physicians are likely to increase, resulting in less time for learning and increasing selectivity in choosing learning activities.

- Professional values and conflicts. Resource limitations will increasingly require physicians to reconcile their roles in working for the best interests of individual patients while keeping in mind the limits on resources available across all patients needing care.

- Personal and professional conflicts. Professional time pressures will increasingly conflict with personal lives. Limits on resources for health care may result in lower net compensation.

\section{Information Systems}

The increasing volume of information is difficult for individual physicians to manage. Opportunities for improving physicians' use of information include the following:

- Identify authoritative information sources. Provide guidance regarding the reliability of information sources. This guidance will help physicians focus their limited time across information sources.

- Prioritize new information. Identify new high-priority information. Specialty societies or other national groups can identify recently published information that has sufficient potential health impact on patients that everyone in a specialty should be aware of and implement it. The group identifying "patient-oriented evidence that matters" (POEMs) has demonstrated the feasibility of identifying high-priority information. ${ }^{22}$
- Link types of information. When presenting biologic information on a topic, also present related psychosocial, organizational, and financial information associated with implementation.

- Accessibility. Use technology to make information sources more easily accessible. This can include access to consultants through electronic mail, information resources on personal handheld devices, wireless networking, and information proactively built into clinical data systems.

\section{Educational Systems}

The increasing volume of new information produces a parallel increase in potential new educational content for physicians. The focus of content tends to be on biologic knowledge, with less attention paid to information related to implementation, in part reflecting a cultural perception in medicine that academic expertise is biologic expertise. Opportunities for improvements in educational systems include the following:

- Prioritize new curricula. Prioritizing new information by specialty (see "Information Systems") would produce periodic "national needs assessments" for learning by specialty. These curricular priorities could help guide all organizations that plan formal educational activities for physicians.

- Integrate content relevant to change. Educational offerings should go beyond new biologic knowledge to include psychosocial, organizational, and financial considerations relevant to actually implementing the information into practice.

- Cost-effective selection of educational methods. The least costly method (e.g., lecture, small-group discussion) to provide necessary learning is likely to depend on the type of learning under consideration (e.g., simple fact in a stable context or complex change in care). The development of a 
classification for learning situations by the likely effectiveness and cost of commonly used educational methods would facilitate curricular design in an environment of constrained resources.

\section{Implementation Systems}

Both the aging population and new information about health care are increasing the amount of health care provided per person. Administrative priorities within health care systems often focus on coordination for selected high-priority activities. Assignments of administrative responsibilities often result in different units having operational responsibility for staff education and for quality assurance and improvement. Opportunities for improvements in implementation systems include the following:

- Cost-effective selection of change methods. Educational methods (e.g., lecture, small group, feedback) are one set of a larger array of methods for implementing change (e.g., guidelines, critical pathways, reminder systems, financial incentives). A classification for types of situations for change needs to be developed that can be related to the likely effectiveness and cost of commonly used change methods.

- Functionally link the education and quality assurance subsystems. A closer working relationship will help identify common clinical issues and how each unit can costeffectively reinforce or expand the efforts of the other.

- Funding for cost-effective changes. Institutions are likely to fund educational initiatives that are part of changes to provide quality care more cost-effectively.

\section{Regulatory Systems}

Increasingly, public and private groups want regulatory bodies to document the adequacy of regulatory oversight. These pressures are usually not accompanied by commensurate increases in funding. The decentralization of the health care system limits the quantity and uniformity of information that regulatory bodies can obtain relevant to the systems under their jurisdiction. Historically, physicians have tended to place a high value on professional independence and local autonomy. Many physicians are uneasy with increased regulation of their activities. Even within these constraints, some opportunities are evident:

- Enhanced technology and data availability. Regulatory bodies can increase their actual oversight through technologic innovations that increase the availability of data from primary sources and decrease the cost of data collection. However, data systems must become more uniform to be broadly useful for regulation. ${ }^{23}$

- Emerging consensus on priorities. As various information, education, and implementation systems evolve clearer national priorities (e.g., core clinical competencies by specialty, most important new information by specialty), regulatory bodies may focus on oversight activities related to these areas.

\section{Broader Implications for CME and Its Future}

The systems-based conceptual framework provides insight into the overall nature of the "change system," general opportunities for improvement, approaches to research, and helpful actions by individuals in the "CME profession."

\section{Nature of the "Change System"}

No simple system for change exists. Several types of systems are part of the overall processes for physician learning in the translation of knowledge into practice. These systems are largely decentralized, reflecting the general decentral- 
ization of the larger health care system. ${ }^{10}$ Each type of system is structured and funded separately, with a system's outputs primarily valued by the extent to which they sustain the producing system. Little structural or economic incentive exists for one type of system to focus on how its outputs will facilitate processes in other types of systems. Relationships do exist between the different types of systems, but little national guidance is provided concerning the nature and priorities for content in these relationships. The overall functioning of loosely linked systems can be viewed with ambivalence: disappointment that new knowledge is not translated into practice speedily and uniformly and appreciation for change occurring to the extent that it does.

No one simple action will radically alter all of the processes across all of these interrelated systems. Various individuals and groups have made useful recommendations for improvement, for example, link information across the continuum of medical education, focus on evidence-based medicine, depend less on lecture formats, use new technology for easier access, facilitate learning in the workplace, and focus on outcomes. When considered in the context of a systems-based framework, either the proposed action is not simple (difficult to do within the existing infrastructure) or, if done within one type of system, would not produce a fundamental change in the overall processes. Specific changes are worthwhile and should be pursued. However, fundamental changes to the overall process are likely beyond unilateral action by any one type of system.

To plan for broader changes, clearer and more consistent terminology should be used when discussing specific processes and the types of systems performing them. The different types of systems often have somewhat different meanings for specific words. One of the most obvious examples is the use of the words "education" and "learning." Sometimes they are used interchangeably. In the conceptual framework in Figure 1, they are used to refer to related but conceptually and operationally different systems and processes. Educa- tion refers to formal curricula and learning activities planned by an expert operating within a system that provides formal education activities. Physician learning occurs within the individual and includes knowledge and skills acquired from a variety of sources, with educational activities being only one source.

\section{Broader Concerns and Opportunities}

An overarching concern is ensuring future sources of funding for all of the processes necessary for translating new information into physicians' practices. Financial pressures in the larger health care environment will affect all of the types of systems represented in Figure 1. Much of the funding for current processes now comes from indirect sources. Surpluses in health care revenue subsidize many of the systems, including the professional time of journal authors and reviewers, faculty presenting CME activities, and volunteers assisting regulatory bodies. Commercial companies with new products provide much of the advertising revenue and other support on which journals and CME activities rely. Reduced profitability for health care and for related products could substantially reduce funding from these sources. ${ }^{24}$ Attempts to revise the functioning and funding of the overall health care system should include explicit funding for the processes for translating new knowledge into physicians' practices.

Activities will increasingly need to be prioritized, linked, and performed more efficiently across all types of systems involved in translating knowledge into physicians' practices. The increasing rate of production of new knowledge is quantitatively increasing the total "throughput" across all systems. An ever-increasing quantitative overload of needed changes in physician's performance cannot be addressed simply by publishing more journals, offering more CME courses, and developing more performance measures. Changes must also occur within and across the various systems that facilitate needed change. Among the broadly needed changes are the following: 
- Envision the entire set of processes. Individual systems should routinely consider how their outputs (e.g., journal articles, CME activities, performance measures) could be redesigned to facilitate their use as inputs by other systems in the overall change process.

- Centralization and standardization. Fiscal constraints, greater oversight, and enhanced technology will increase pressures for more efficient processes within and between systems. The efficiencies of centralization and standardization across systems will have to be balanced with the flexibilities of decentralization and local variation.

- Priorities. Time and resources are limited. Priorities for change need to be identified and coordinated across systems.

- All of the needed types of information. Many systems currently focus on biologic information. They need more adequately to address related psychosocial, operational, and financial issues associated with implementing new biologic information.

- Ensure adequate expertise. Systems-particularly educational systems-generally need to expand their access to expertise in psychosocial, operational, and financial issues associated with recommended biologic changes. Formal efforts may be needed to develop, recognize, and reward these types of expertise.

- Cost-effective methods. All of the factors to be addressed in implementing a change should be considered in selecting a method or combination of methods for change that will likely be cost-effective in producing change.

- Demonstrate value. As resources within the overall health care system become more constrained, all systems in it will be increasingly pressed to demonstrate that the benefits they produce are worth the costs. Libraries are deciding which journal subscriptions to renew, physicians which CME courses to attend, employers which health care plan to purchase, and physicians whether to recertify.

The following examples illustrate the use of a systems-based perspective to enhance performance. The first example illustrates an approach to increase the likelihood that CME activities will facilitate improved performance. The Accreditation Council for Continuing Medical Education (ACCME) currently addresses performance outcomes only by recognizing "exemplary compliance" for evaluation if a provider measures practice application or health status improvement. ${ }^{25}$ Many CME providers are not directly linked to health care implementation systems, making measurement of actual performance unlikely. ACCME provides these CME providers with no other incentive to facilitate actual performance. ACCME could also recognize providers who have curricula that systematically address the information needed to implement recommended changes in the practice settings of the intended audience. Achieving this recognition for facilitating performance outcomes would be reasonably possible for all CME providers.

The second example illustrates how different types of systems could work together to increase efficiency in ensuring that high-priority information is recognized and implemented. CME providers, residency program directors, and others now independently identify important new information for physicians in a specialty. A medical specialty could develop a process to identify new information that, based on likely patient impact, was a high priority for all physicians in the specialty to know and implement. When initially identified, the information would be a priority for CME curricula and for inclusion in the core knowledge associated with competencies taught in residency programs. With the passage of time for incorporation into education and practice, the high-priority information would be a likely source used in designing reviews of residency programs, competency assessments for practicing physicians, and board review examinations for the specialty. 


\section{Implications for Research}

The conceptual framework in Figure 1 can help researchers understand how to integrate strengths from different literature and guide researchers' selection of problems likely to have a significant impact.

Different types of systems in Figure 1 have developed somewhat different interests in research. Research literatures in CME, in health care quality assurance and improvement, and in physician behavior change are important to addressing the overall processes. Although these literatures share theory, methods, and aims, each has evolved somewhat unique emphases. With a great deal of oversimplification, some general comparisons are as follows:

- $C M E$. Originating primarily from work in education systems and in physician learning, this literature tends to emphasize the individual's learning processes and motivations and the active role of the individual physician. This literature draws heavily on psychological theories. The literature comes from an academic culture that values "doing the right thing." A frequent limitation is that effectiveness is studied, but costs and cost-effectiveness are not.

- Quality assurance and improvement. Originating primarily from work within implementation systems, this literature tends to emphasize local problem solving in realworld situations within an institution. It draws less on conceptual theory than on individuals applying procedural steps of problem analysis, consideration of options, selecting and implementing a solution, and evaluating the result. The literature comes from an operational culture that values practicality. Although this approach applies local solutions to issues, a frequent limitation is that it provides little conceptual insight about general principles likely to be useful across situations or institutions. ${ }^{26}$
- Physician performance change. This literature tends to be a subset of the health services research literature. Originating from work within and across several types of systems, it emphasizes organizational and structural approaches to methods for change (e.g., guidelines, education, reminders, financial incentives). The literature comes from an administrative culture that values cost-effective planning. A frequent limitation is an oversimplification of underlying processes in specific settings. For example, change methods (e.g., reminders) tend to be discussed as though they are a uniform intervention rather than one aspect of a complex process that can have different implications in different contexts.

The strengths from these three research traditions need to be better integrated into performing research on the processes that translate new knowledge into physicians' practices. The learning processes and motives of physicians, the cultural and operational contexts of the local institution, and the ability to generalize cost-effective methods for change are all important in the overall framework. Although thoughtful examples of integration can be found, ${ }^{27}$ researchers tend to stay within the traditions within which they were trained and publish.

Researchers can use the list of broader opportunities for improvement (presented earlier) for guidance as they consider how to frame research questions and design research projects. The research implications of three items from the list are further considered here.

Priorities. The larger systems-based framework should help prioritize research topics for investigation. How much impact is the study likely to have on the actual performance of important aspects of care? For example, although the educational literature includes many studies of personal learning styles, the ultimate value of detailed study of learning styles of physicians may not be great. Physi- 


\section{Lessons for Practice}

- Consider how you can increase your effectiveness within the larger framework for translating new knowledge into physicians' practices:

- How can you work more effectively within your own system?

- How can systems that provide input to you do so more effectively?

- How can you change your work outputs to be more effective for those who use them in their systems?

- Research and practice to improve physician performance should integrate the strengths of theoretical and research literature addressing $\mathrm{CME}$, quality improvement, and physician performance change.

cians are a highly selected subset of the population that have demonstrated, through medical school and residency, their ability to deal successfully with information presented in a variety of common formats, whether or not personally preferred.

Classification of change situations. A high priority for research is the development and validation of a system to classify change situations according to the types of interventions likely to be effective and cost-effective. Reviews of the effectiveness of intervention methods (e.g., education, feedback) to improve physician performance have found some methods to be effective more often than others. ${ }^{8,12}$ However, none of the methods work consistently or consistently do not work. Identifying the underlying circumstances that enable a method to be successful will greatly advance practice. The systems-based approach used for Figure 1 provides a basis to begin to identify a sequence of factors to consider and which ones--singly or in combination--are necessary for changing performance related to a specific aspect of care. More elaborate conceptual frameworks have been developed that could be applied to research on this issue. $17,27,28$

Cost-effectiveness. With expected financial constraints on the health care system, research on translating new knowledge into physicians' practices should also measure the costs of change efforts and project likely cost-effectiveness. ${ }^{29,30}$ Policy makers need this information when deciding whether applied research or evaluation findings should be the basis for broader changes.

\section{Implications for CME Professionals}

Individuals working in the CME system can use the preceding systems-based analysis to develop insights into their roles and their professional futures:

- Your role. Use the broader conceptual framework to understand your role in the overall processes and outcomes desired.

- Improve your effectiveness. Consider how you can work more effectively within your own system. Also, consider how you might work toward having systems that provide input to you do so in ways that make you more effective.

- Improve the effectiveness of others. Consider how your work outputs can be improved to make others who use them more effective in their work.

- Plan for change. Societal pressures will result in an ongoing series of changes in all types of systems involved in the translation of new knowledge into physicians' practices. In addition to addressing changes in your direct work, monitor changes and their implications in systems that provide input to you and systems to which you provide output. 
- Communicate clearly. When discussing issues and proposed changes, use a systems-based vocabulary to communicate clearly your ideas and intentions regarding specific activities.

- Cost and cost-effectiveness. Recognize the costs and the cost-effectiveness of your work activities to identify ways to ensure that higher-priority activities continue to be performed as financial limitations increase.

\section{References}

1. National Center for Health Statistics. Health, United States 2002, with chartbook on trends in the health of Americans: Table 117. Hyattsville, MD: National Center for Health Statistics, 2002.

2. Committee on the Quality of Health Care. Crossing the quality chasm: a new health system for the 21 st century. Washington, DC: National Academy Press, 2001.

3. Bennett NL, Davis DA, Easterling WE, et al. Continuing medical education: a new vision of the professional development of physicians. Acad Med 2000; 75(12):1167-1172.

4. Clancy C. Commentary: reinventing continuing medical education. BMJ 2004; 328:E291.

5. Cohen JJ. A word from the president: transforming CME. AAMC Reporter 2002;(October):2.

6. Council of Medical Specialty Societies. Repositioning for the future of continuing medical education. Lake Bluff, IL: Council of Medical Specialty Societies, 2002.

7. Leist JC, Green JS. Congress 2000: a continuing medical education summit with implications for the future. J Contin Educ Health Prof 2000; 20:247-251.

8. Mazmanian PE, Davis DA. Continuing medical education and the physician as a learner: guide to the evidence. JAMA 2002; 288(9):1057-1060.

9. Whitcomb MF. CME reform: an imperative for improving the quality of medical care. Acad Med 2002; 77(10):943-944.
10. Martin JB, Alpern RJ, Betz AL, et al. Educating doctors to provide high quality medical care: a vision for medical education in the United States. Washington, DC: Association of American Medical Colleges, 2004.

11. Davis DA, Barnes BE, Fox R. The continuing professional development of physicians. Chicago: AMA Press, 2003.

12. Grimshaw JM, Thomas RE, MacLennan G, et al. Effectiveness and efficiency of guideline dissemination and implementation strategies. Health Technol Assess 2004; 8(6):1-72.

13. Berwick DM. Disseminating innovations in health care. JAMA 2003; 289(15):1969-1975.

14. Grol R. Improving the quality of medical care: building bridges among professional pride, payer profit, and patient satisfaction. JAMA $2001 ; 286(20): 2578-2585$.

15. Miller JG. Living systems. New York: McGraw-Hill, 1978.

16. Katz D, Kahn RL. The social psychology of organizations. New York: John Wiley \& Sons, 1978.

17. Cabana MD, Rand CS, Powe NR, et al. Why don't physicians follow clinical practice guidelines? A framework for improvement. JAMA 1999; 282(15):1458-1465.

18. Pathman DE, Konrad TR, Freed GL, Freeman VA, Koch GG. The awareness-to-adherence model of the steps to clinical guideline compliance: the case of pediatric vaccine recommendations. Med Care 1996; 34(9):873-889.

19. Burney RE, Harrison RV. Continuing education for practicing surgeons. In: Mulholland $\mathrm{M}$, ed. Complications in surgery. Philadelphia: Lippincott Williams \& Wilkins, 2005. (In press)

20. Deming WE. Out of the crisis. Cambridge, MA: Massachusetts Institute of Technology, Center for Advanced Engineering Study, 1986.

21. Chernew ME, Hirth RA, Cutler DM. Increased spending on health care: how much can the United States afford? Health Affairs 2003; 22(4): 15-25.

22. Slawson DC, Shaughnessy AF. Becoming an information master: using POEMs to change practice with confidence. Patient-oriented evi- 
dence that matters. J Fam Pract 2000; 49(1):63-67.

23. Landon BE, Normand ST, Blumenthal D, Daley J. Physician clinical performance assessment: prospects and barriers. JAMA 2003; 290(9):1183-1189.

24. Harrison RV. The uncertain future of continuing medical education: commercialism and shifts in funding. J Contin Educ Health Prof 2003; 23(4): 198-209.

25. The ACCME's essential areas and their elements, including standards for commercial support. Chicago: Accreditation Council for Continuing Medical Education, 1999.

26. Mittman BS. Creating the evidence base for quality improvement collaboratives. Ann Intern Med 2004; 140:897-901.
27. Grol R. Changing physicians' competence and performance: finding the balance between the individual and the organization. J Contin Educ Health Prof 2002; 22(4):244-250.

28. Walsh JM, McPhee SJ. A systems model of clinical preventive care: an analysis of factors influencing patient and physician. Health Educ Q 1992; 19(2):157-175.

29. Mason J, Freemantle N, Nazareth I, Eccles M, Haines A, Drummond M. When is it cost-effective to change the behavior of health professionals? JAMA 2001; 286(23):2988-2992.

30. Brown CA, Belfield CR, Field SJ. Cost effectiveness of continuing prefessional development in health care; a critical review of the evidence. BMJ 2002; 324:652-655. 